\title{
-Photogravure-
}

\section{Demonstration of Microvessel Networks and Endothelial Cell Phenotypes in the Normal Murine Lung}

Clare Minton¹, Enjing Jin², Namiko Taniuchi², Toshiaki Matsuoka², Shigeki Yamagishi², Mohammad Ghazizadeh ${ }^{2}$ and Oichi Kawanami ${ }^{2}$

${ }^{1}$ Visiting High-school Student, Uppingham School, Uppingham, UK

${ }^{2}$ Department of Molecular Pathology, Institute of Gerontology, Nippon Medical School, Graduate School of Medicine
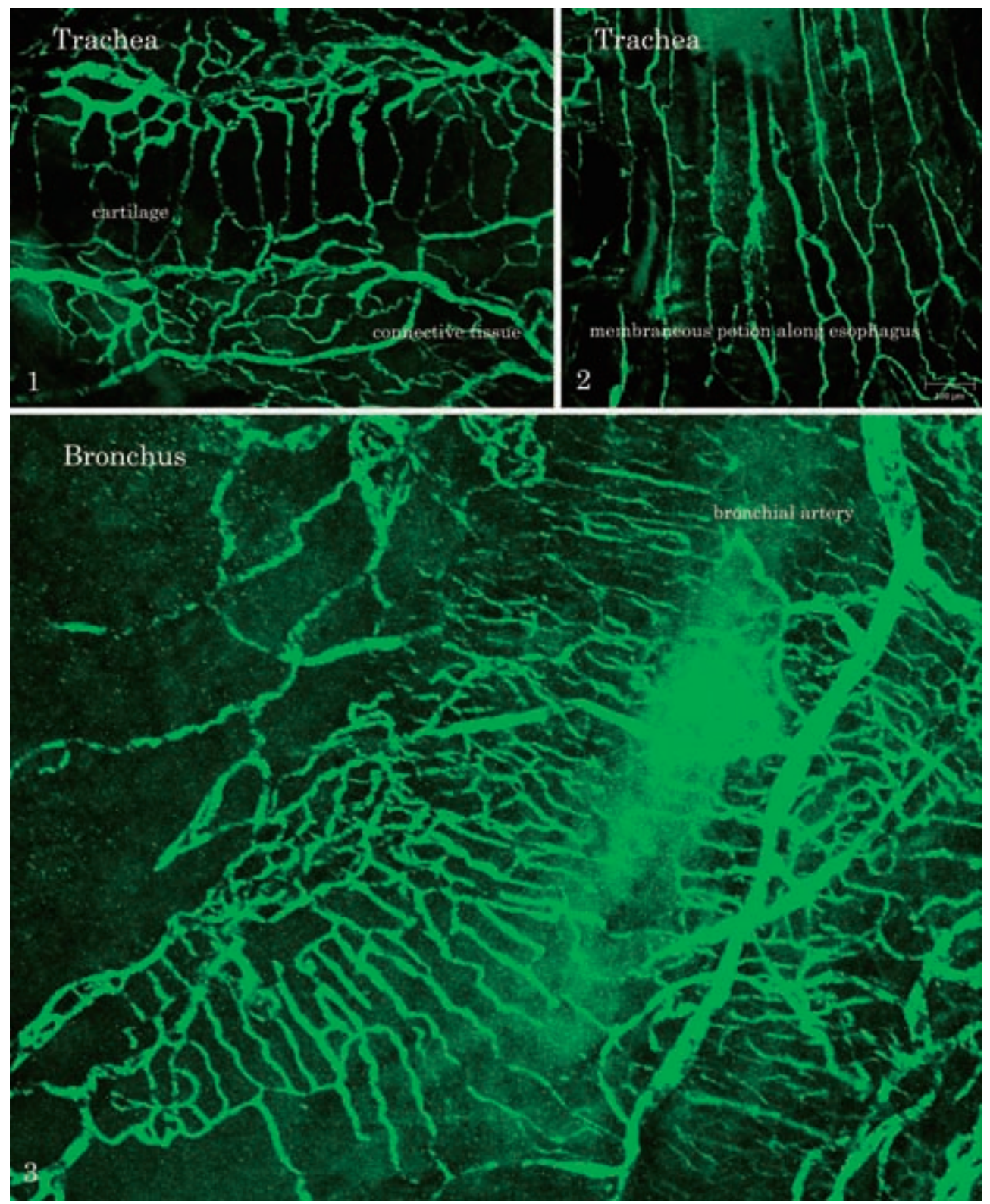

Correspondence to Oichi Kawanami, Department of Molecular Pathology, Institute of Gerontology, Nippon Medical School, 1-396 Kosugi, Nakahara-ku, Kawasaki, Kanagawa 211-8533, Japan

E-mail:kawanami@nms.ac.jp

This work was partly supported by Grants-in-Aid from the Ministry of Education, Culture, Sports, Science and Technology of Japan: \#16790857 (Research for Young Investigator B) and \#15659199 (Exploratory Research). Journal Website (http://www.nms.ac.jp/jnms/) 

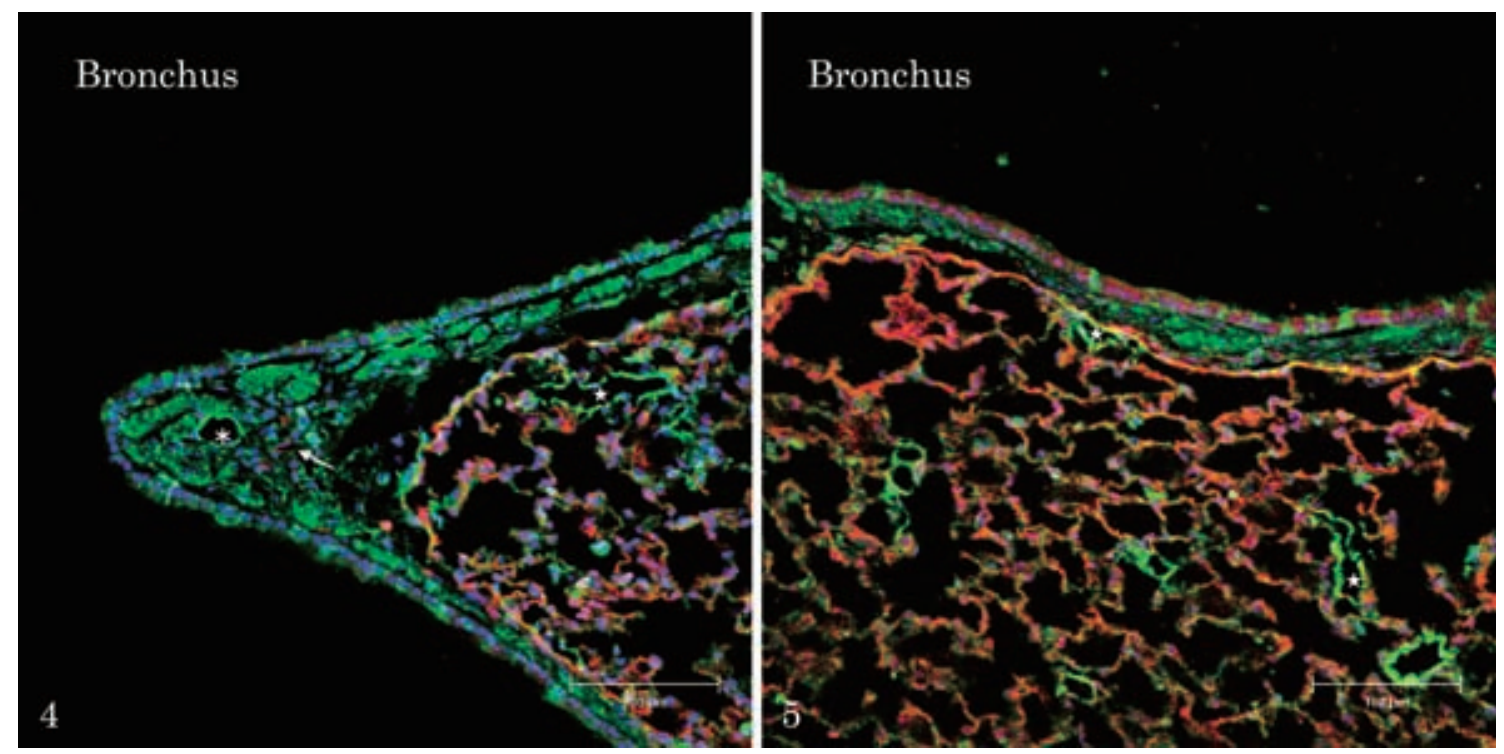

\begin{abstract}
The vascular endothelial cells (ECs) express various antigens related to coagulation factors, including factor VIII-related antigen or von Willebrand factor (vWF) in the cytoplasm and thrombomodulin (TM; a thrombin receptor) along the plasma membrane. CD34 (a hematogenic stem cell marker) is also expressed along the surface membrane of the ECs. Using these EC markers and fluorescein-isothiocyanate-labeled dextran (FITC-dextran) (Sigma Co., St. Louis, MO), we attempted to demonstrate the complex network of microvessels and their EC phenotypes in tracheo-bronchial trees and lung parenchyma of the normal adult ICR male mice.

Under anesthesia, saline with heparin was infused slowly through left ventricle to drain off the blood. Following brief fixation with $4 \%$ buffered paraformaldehyde solution (PFA) through the same route, one group of animals received,

1) FITC-dextran injection via left ventricle, and the large airways and lungs were further fixed in PFA, or 2) The airways and lungs of the other group were rapidly frozen, and the thin sections were stained with two antibodies of vWF and Alexa Fluor 594-labeled CD34. The vWF antibody was later labeled by FITC.

The microvessels of airways and lungs were observed by a laser scanning confocal microscope (TC-SP, Leica, Heidelberg, Germany). The phenotypic characteristics of microvessel ECs appeared mostly identical with those described previously in the human lun $\mathrm{g}^{1,2}$, although CD34 was applied instead of TM in the present study. The topographical heterogeneity of immunohistochemical properties of ECs would suggest functional differences at different sites of the lung, that would provide a novel insight for understanding the pathogenesis of human lung diseases.
\end{abstract}

【A】 FITC-labeled dextran infusion into the tracheobronchial vessels

Fig. 1 Trachea: Capillaries were arranged in parallel array on the tracheal cartilage rings, and converged into polygonal capillary meshwork in the intercartilage connective tissues. They were connected to venules which ran through intercartilage soft tissues.

Fig. 2 Trachea membranous part: Capillaries were arranged parallel to the longitudinal axis of trachea. They occasionally made anastomosis with adjacent vascular branches. (bar: $100 \mu \mathrm{m}$, same in Fig. 1 and 3)

Fig. 3 Bronchus: Subepithelial capillaries were arranged in a ring-like or a parallel pattern. Note the dense meshwork of capillaries that were connected to larger vessels, possibly bronchial arteries that ran obliquely along the longitudinal axis of bronchial trees.

【B】 Dual immunofluorescence staining of microvessel ECs in lung frozen sections

Fig. 4, 5 Bronchus: Only a few microvessels (star in Fig. 4) were located in scarce amount of peribronchial soft tissue, showing positive reaction to vWF (FITC green color) alone. Subepithelial capillaries (arrow) were reactive to CD34 (Alexa Fluor 594 red color), but not to vWF. The pulmonary venules or arterioles (stars in Fig. 4 and 5) located among alveolar capillaries were reactive mainly to $\mathrm{vWF}$, and partial reaction to CD34 along the microvessel loops clearly demonstrates a mosaic-like staining pattern. The alveolar capillary ECs were exclusively reactive to CD34, but totally unreactive to vWF. Therefore, the lung microvessels comprise distinct phenotypes of ECs with topographic differences.

\title{
References
}

1. Kawanami O, Jin E, Ghazizadeh M, Fujiwara M, Jiang L, Ohaki Y, Gomibuchi M, Takemura T: Mosaiclike distribution of endothelial cell antigens in capillaries and juxta-alveolar microvessels in the normal human lung. Pathol Int 2000; 50: 136-141.

2. Jin E, Kawanami O: Unique distribution of von Willebrand factor and thrombomodulin in endothelial cells of human pulmonary microvessels. J Nippon Med Sch 2000; 67: 64-65. 